\title{
Aggressive breast fibromatosis with axillary accessory breast mastitis mimicking carcinoma: a case report
}

\author{
Yuhong Huang ${ }^{1}$, Nan Shao ${ }^{1}$, Huijuan Shi ${ }^{2}$, Xiaoling Zhang ${ }^{3}$, Yanling Zheng ${ }^{4}$, Ying Lin ${ }^{1}$ \\ ${ }^{1}$ Breast Disease Center, The First Affiliated Hospital, Sun Yat-sen University, Guangzhou, China; 2 Department of Pathology, The First Affiliated \\ Hospital, Sun Yat-sen University, Guangzhou, China; ${ }^{3}$ Department of Radiology, The First Affiliated Hospital, Sun Yat-sen University, Guangzhou, \\ China; ${ }^{4}$ Department of Medical Ultrasonics, Institute of Diagnostic and Interventional Ultrasound, The First Affiliated Hospital, Sun Yat-sen \\ University, Guangzhou, China \\ Correspondence to: Ying Lin. Breast Disease Center, The First Affiliated Hospital of Sun Yat-sen University, No. 58 Zhongshan Road 2, Guangzhou, \\ China. Email: linying3@mail.sysu.edu.cn.
}

\begin{abstract}
Aggressive fibromatosis, also referred to as desmoid-type fibromatosis or desmoid tumor, is an uncommon locally invasive tumor with a high local recurrence rate. It rarely occurs in the breast and the etiology of aggressive breast fibromatosis is still unknown but may be related to previous mammary trauma or surgery. Preoperative diagnosis of aggressive breast fibromatosis is still a challenge for clinicians. The optimal treatment for the disease is still controversial, but wide surgical excision with negative margins is considered the standard option. We report a 28-year-old woman of aggressive breast fibromatosis accompanied with accessory breast mastitis in her ipsilateral axilla. The lesions imitated breast carcinoma with axillary lymph node metastasis due to their similar clinical and imaging manifestations. Her mammary mass finally underwent wide surgical excision, and negative margins were confirmed during the surgery. To our knowledge, this is an extremely rare case accompanied with axillary accessory breast mastitis and will contribute to the management of aggressive breast fibromatosis.
\end{abstract}

Keywords: Breast; aggressive fibromatosis; axilla; accessory breast; mastitis; case report

Received: 25 July 2020; Accepted: 10 September 2020; Published: 31 January 2021.

doi: 10.21037/tbcr-20-41

View this article at: http://dx.doi.org/10.21037/tbcr-20-41

\section{Introduction}

Aggressive fibromatosis, also called desmoid tumor or desmoid-type fibromatosis, is an uncommon benign tumor originating from fibroblasts and myofibroblasts of deep soft tissue $(1,2)$. The tumor is characterized by slow growth, local invasion, and high recurrence rate, but no tendency of distant metastasis. Aggressive fibromatosis could occur in the abdominal wall, intra-abdominal region, and extraabdominal region. According to reported cases, aggressive breast fibromatosis was sporadic, accounting for fewer than $10 \%$ of all aggressive fibromatosis $(3,4)$. The etiology of aggressive breast fibromatosis remains uncertain, but the disease may be related to previous trauma, surgery (especially breast augmentation), and some inherited diseases (such as familial adenomatous polyposis). Its incidence accounts for $0.2 \%$ of all primary breast neoplasm, and the local recurrence rate is about $29 \%$, mostly occurring within three years after surgery $(3,5,6)$.

Clinically, aggressive breast fibromatosis usually presents as a palpable isolated painless firm mass that may attach to the chest wall, and usually causing dimpling or retraction of the skin, and neither nipple discharge nor axillary lymphadenopathy is common (1). The mass is easy to be misdiagnosed as breast carcinoma as its margins are usually unclear due to tumor local infiltration. In imaging findings, it is characterized as an irregular mass with high density, and sometimes with the burr-like edges, but without calcification, thus the lesion may be described as breast carcinoma (7). Preoperative biopsy examination provides pathological detail of the mass and usually plays a more critical role than images. Microscopically, the 

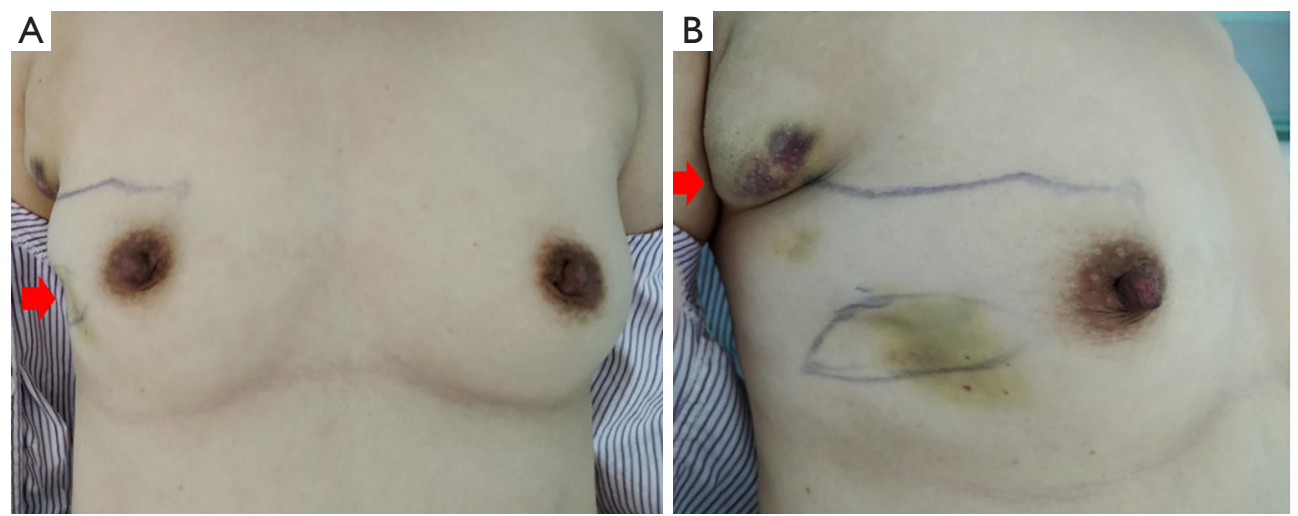

Figure 1 Physical examination of the case showed (A) dimple sign (arrow) of right breast and (B) ipsilateral axillary lesion (arrow).

tumor is composed of long, sweeping and interlacing fascicles of spindle cells with hypochromatic, without atypia or mitotic activity, often infiltrating adjacent epithelium and stroma. The differential diagnosis of aggressive breast fibromatosis is challenging, includes neoplastic spindle cell proliferations, such as fibromatosislike spindle cell metaplastic carcinoma, cystosarcoma phylloides, fibrosarcoma and myofibroblastic tumor and benign lesions such as radial scars and nodular fasciitis. Immunohistochemistry method including SMA, $\beta$-catenin (nuclear), CD34, S-100 protein and cytokeratins can help to make the differential diagnosis. The most crucial differential is with fibromatosis-like spindle cell metaplastic carcinoma, and nuclear atypia and an elevated mitotic rate could help to differentiate from aggressive fibromatosis. To distinguish aggressive fibromatosis from fibrosarcoma, the absence of nuclear pleomorphism and high mitotic index in microscopy are helpful $(1,8,9)$.

Reported treatments to aggressive breast fibromatosis include surgical excision, radiotherapy and kinds of medications. Preoperative pathological diagnosis and wide surgical excision with negative margins are of vital importance for aggressive breast fibromatosis (4). We encountered an extremely rare case of aggressive breast fibromatosis accompanied with axillary accessory breast mastitis, which was more easily misdiagnosed as breast carcinoma with axillary lymph node metastasis clinically than other cases. The axillary lesion increased the difficulty in clinical diagnosis to aggressive breast fibromatosis preoperatively. We present the following article in accordance with CARE Checklist (available at http://dx.doi. org/10.21037/tbcr-20-41).

\section{Case presentation}

In November 2019, a 28-year-old woman came to our hospital because of palpable masses in her right breast and axilla for about one year. She had been treated by traditional Chinese medicine (TCM) treatment for about ten months. The patient underwent axillary minimally invasive surgery because of axillary hyperhydrosis two years ago. The patient's menstrual cycle was regular, and she denied any family history of carcinoma or inherited diseases. She had not taken oral contraceptives, drunk or smoke, and there was no history of mammary trauma or surgery.

Physical examination revealed a firm, painless mass measuring $2.5 \mathrm{~cm} \times 3.0 \mathrm{~cm}$ in the lower outer quadrant of the right breast with dimpling sign, and there was no nipple discharge (Figure 1A). One enlarged painless lymph node measuring $1.0 \mathrm{~cm} \times 2.0 \mathrm{~cm}$ could be palpated in her right axilla (Figure $1 B$ ).

In January 2019, the ultrasound examination in the local hospital showed no nodule in her right breast, but two axillary regular homogeneous hypoechoic nodules which were considered enlarged lymph nodes, and then she started TCM treatment. During treatment, a mass in the lower outer quadrant of right breast was noted. Came to our hospital, her ultrasound showed a solid heterogeneous hypoechoic irregular mass measuring about $1.0 \mathrm{~cm} \times$ $1.9 \mathrm{~cm}$ at 8 o'clock of her right breast (Figure 2). Ipsilateral axillary ultrasound showed three regular homogeneous hypoechoic nodules measuring $0.5-1.7 \mathrm{~cm}$ in size (Figure 2C,D). The ultrasound report classified the mammary mass into BI-RADS 5 and it was considered breast carcinoma with axillary lymph node metastasis. Her 

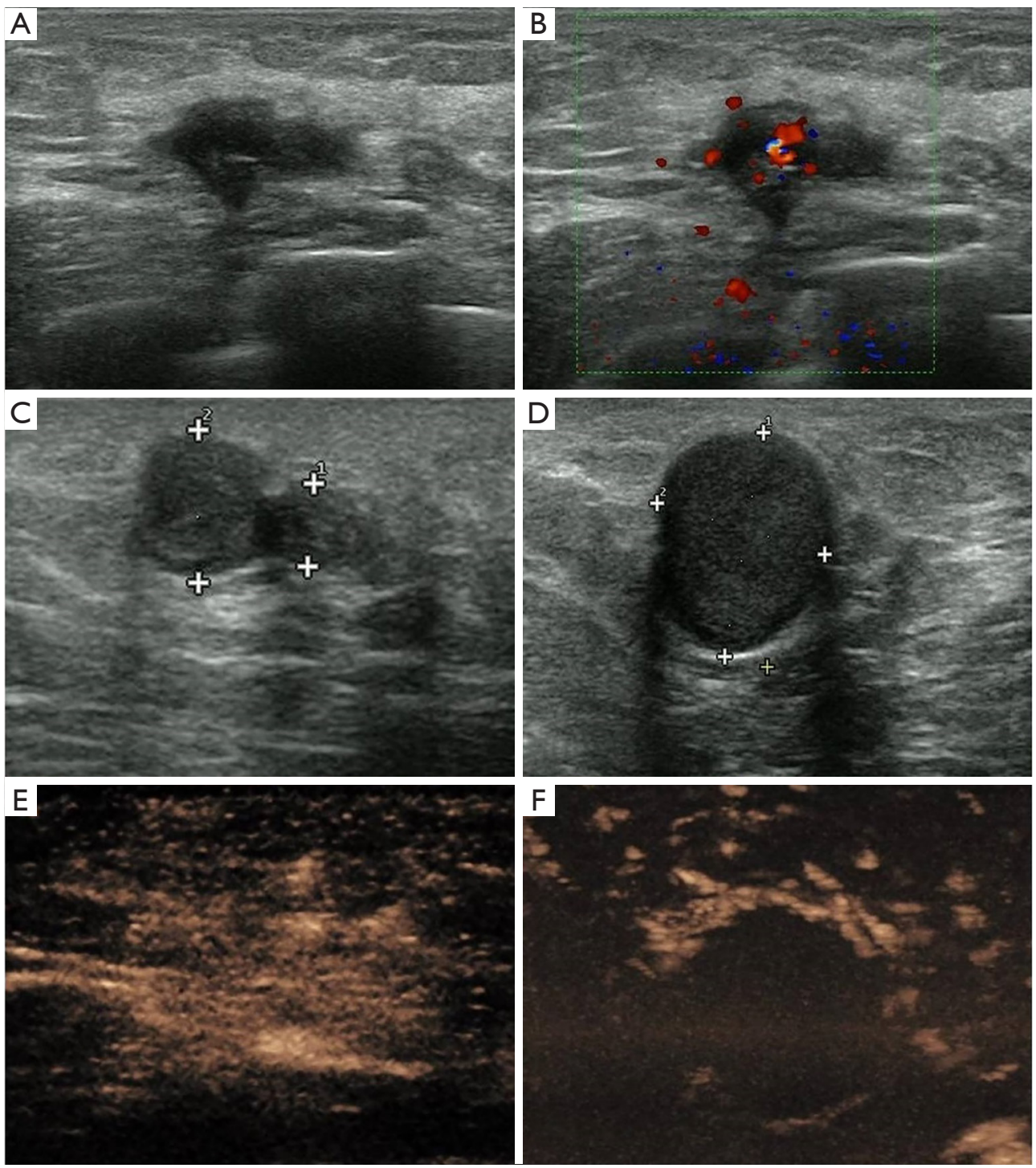

Figure 2 Ultrasound images of right breast and axilla. (A) A hypoechoic nodule with irregular margins and (B) abundant blood supply inside was showed. (C,D) Three round hypoechoic nodules without valve structure were detected. After injection of contrast agent, (E) the mammary nodule showed uneven enhancement with irregular edge and (F) the axillary nodules showed no enhancement inside.

mammograms showed no mass or calcification in the breast (Figure 3A,B). However, dense breast parenchyma may cause the mass inadequately visualized on mammograms in some patients.

More examinations for the patient were done after her admission into our hospital. Significantly, the contrastenhanced ultrasound revealed an irregular enhanced mass which was $1.7 \mathrm{~cm} \times 2.0 \mathrm{~cm} \times 2.6 \mathrm{~cm}$ in her right breast (Figure 2E). That was considered as breast carcinoma which was classified into BI-RADS 4c. However, there was no enhancement in her axilla (Figure 2F). The DCEMRI (dynamic contrast-enhanced magnetic resonance image) showed an irregular $1.6 \mathrm{~cm} \times 1.7 \mathrm{~cm}$ mass that invading the chest wall in the lower outer quadrant of the right breast (Figure 4A,B). The DCE-MRI did not reveal any lesion in her axilla. Fluorodeoxyglucose positron emission tomography/computed tomography (FDG$\mathrm{PET} / \mathrm{CT}$ ) showed that in the right breast, a mass was inhomogeneous with increased standard uptake value (SUV) and the max value was 2.90 (Figure $5 A, B$ ) and the 

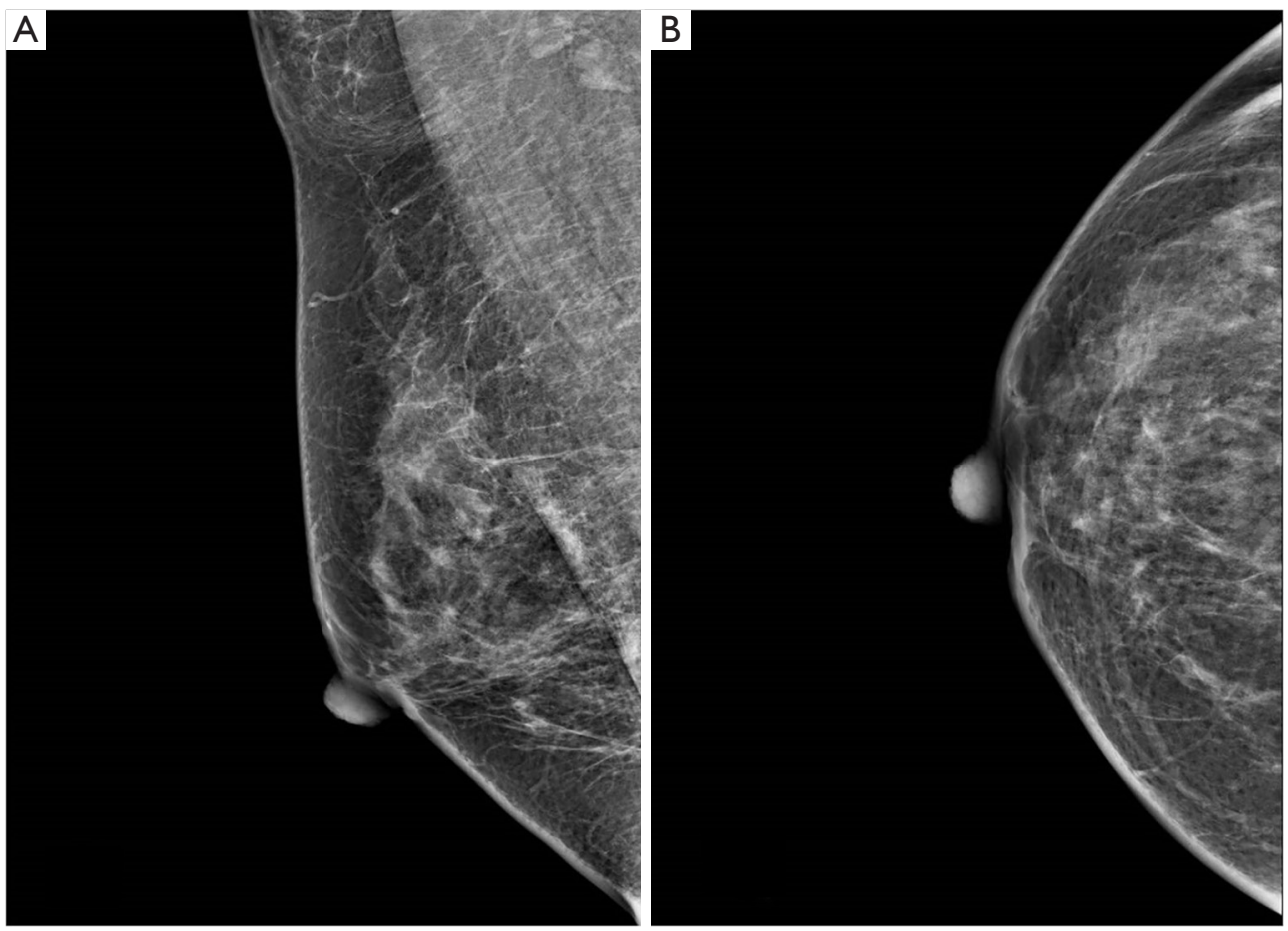

Figure 3 Mammography of the right breast. No abnormal findings in (A) mediolateral oblique view and (B) craniocaudal view.
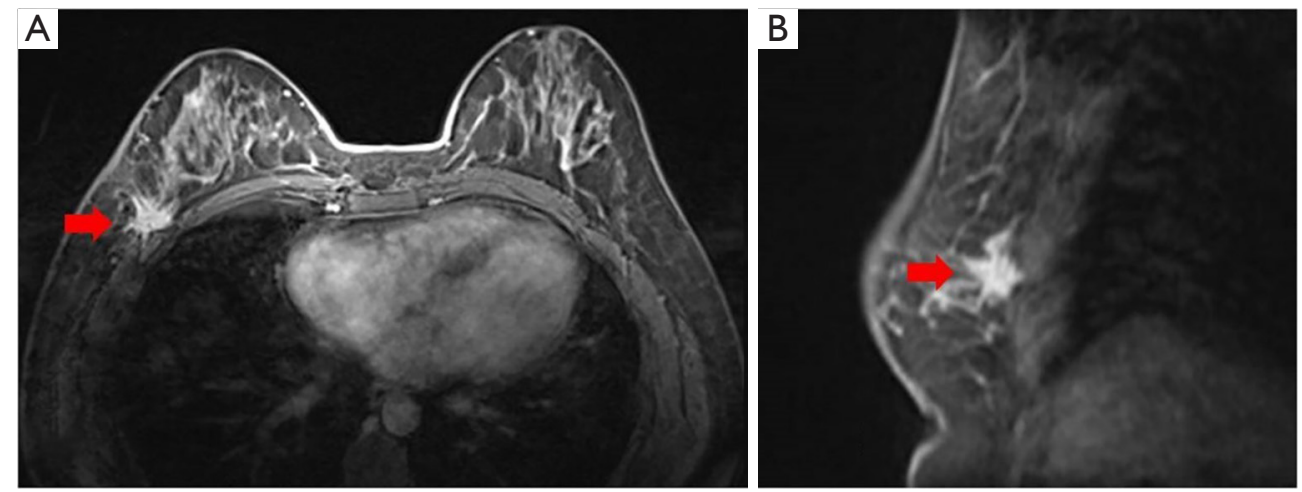

Figure 4 Dynamic contrast-enhanced MRI of the breast. Contrast-enhanced T1 image demonstrated a mass (arrow) attached to the right chest wall in (A) axial incision and (B) sagittal incision.

mass had a clear boundary with chest wall. Moreover, a dense mass in the right axilla was noted and the max SUV was 2.30. Ultrasound-guided biopsy to the mammary mass demonstrated the proliferation of spindle cells (Figure $6 A$ ) with positive immunohistochemical staining for $\beta$-catenin and only scattered cells positive for $\mathrm{p} 63$, but negative immunohistochemical staining for CK5/6, CK7, and CK (HMW). It supported the preoperative diagnosis of aggressive breast fibromatosis. Also, the biopsy of axillary masses revealed no tumor cells but focal foam cells (Figure $6 B$ ) with positive immunohistochemical staining for CD68, but negative immunohistochemical staining for CK. As such a disease is rare and the local recurrence rate after surgery is high, so our patient was discussed at the multi-disciplinary consultation, and surgical intervention was recommended. Finally, the patient agreed to breastconserving surgery and sentinel lymph node biopsy.

The patient underwent surgical excision of the 
A

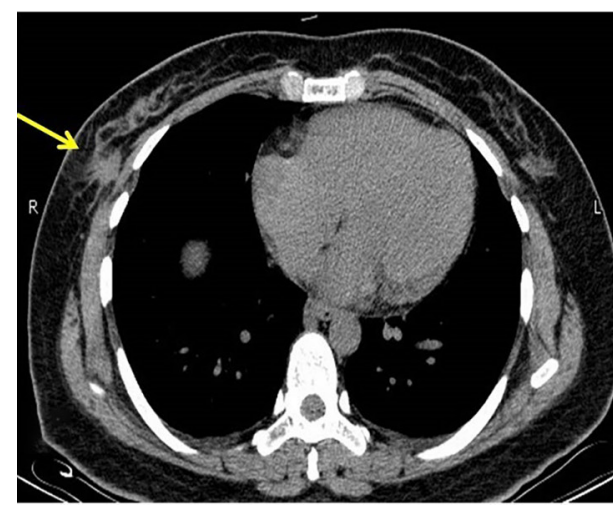

B

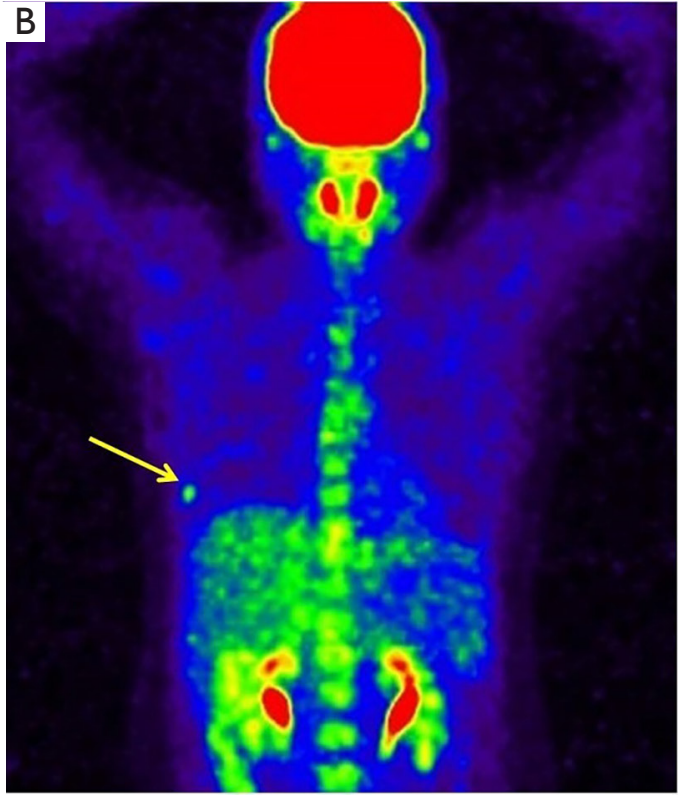

Figure 5 Imaging of 18 F-fluorodeoxyglucose (FDG) PET/CT. (A) Transverse section of CT plain scan revealed an irregular mass (arrow) with spiculated margin and (B) saccharometabolism image showed an abnormal mass (arrow).

mammary mass and the axillary modules, and postoperative pathological diagnosis was made (Figures 6,7). During surgery, about $3.0 \mathrm{~cm} \times 4.0 \mathrm{~cm}$ pectoralis major muscle under the mass was resected for the mass could not be easily separated from adjacent pectoralis major muscle. The wide surgical resection was completed, and all margins of the surgical cavity were free of tumor. In the right axilla, we observed that the anatomical layers were difficult to distinguish, and some caseous lesions was noted (Figure 7A). In addition, the intraoperative pathological result suggested no tumor cell in the sentinel lymph node (0/2) (Figure 6C).

A definitive diagnosis of aggressive breast fibromatosis was established from the final resection pathology. Postoperative resection pathology showed the mammary mass mainly composed of fascicular-arranged mildly atypical spindle cells (Figure 6F). The immunohistochemical staining of tumor cell was positive for $\beta$-catenin and CK (focal expression), and negative for CK5/6, CK (HMW), p63, and $\mathrm{CD} 34$, and the $\mathrm{Ki}-67$ was estimated as $8 \%$. The result supported the diagnosis of aggressive breast fibromatosis. Axillary pathology demonstrated accessory breast mastitis according to foam cells and chronic inflammatory cells infiltrating inside (Figure 6E), and the axillary lymph nodes were free from tumor metastasis (Figure 6D). After surgery, the patient was at ease to the negative margins and we advised her that breast ultrasound should be followed up at six-month intervals for at least three years, and she agreed to follow our advice to ensure a good prognosis. The patient is followed now without recurrence for half a year. All procedures performed in studies involving human participants were in accordance with the ethical standards of the institutional and/or national research committee(s) and with the Helsinki Declaration (as revised in 2013). The patient has given the consent for the case report to be published.

\section{Discussion}

We reported an extremely rare case of aggressive breast fibromatosis accompanied with axillary accessory breast mastitis. There is no history of mammary surgery or trauma for the patient. At the first time when she touched the axillary masses, she went to the local hospital, and the ultrasound examination reported two axillary masses but no mammary mass. Then she started her TCM treatment for about ten months.

Aggressive fibromatosis is defined as an intermediate soft-tissue tumor that is characterized by monoclonal proliferation of fibroblast or myofibroblast $(1,2)$. The tumor has a locally invasive tendency and a high incidence of local recurrence but has no potential to metastasize. The incidence rate of aggressive fibromatosis is 2.4-4.3 

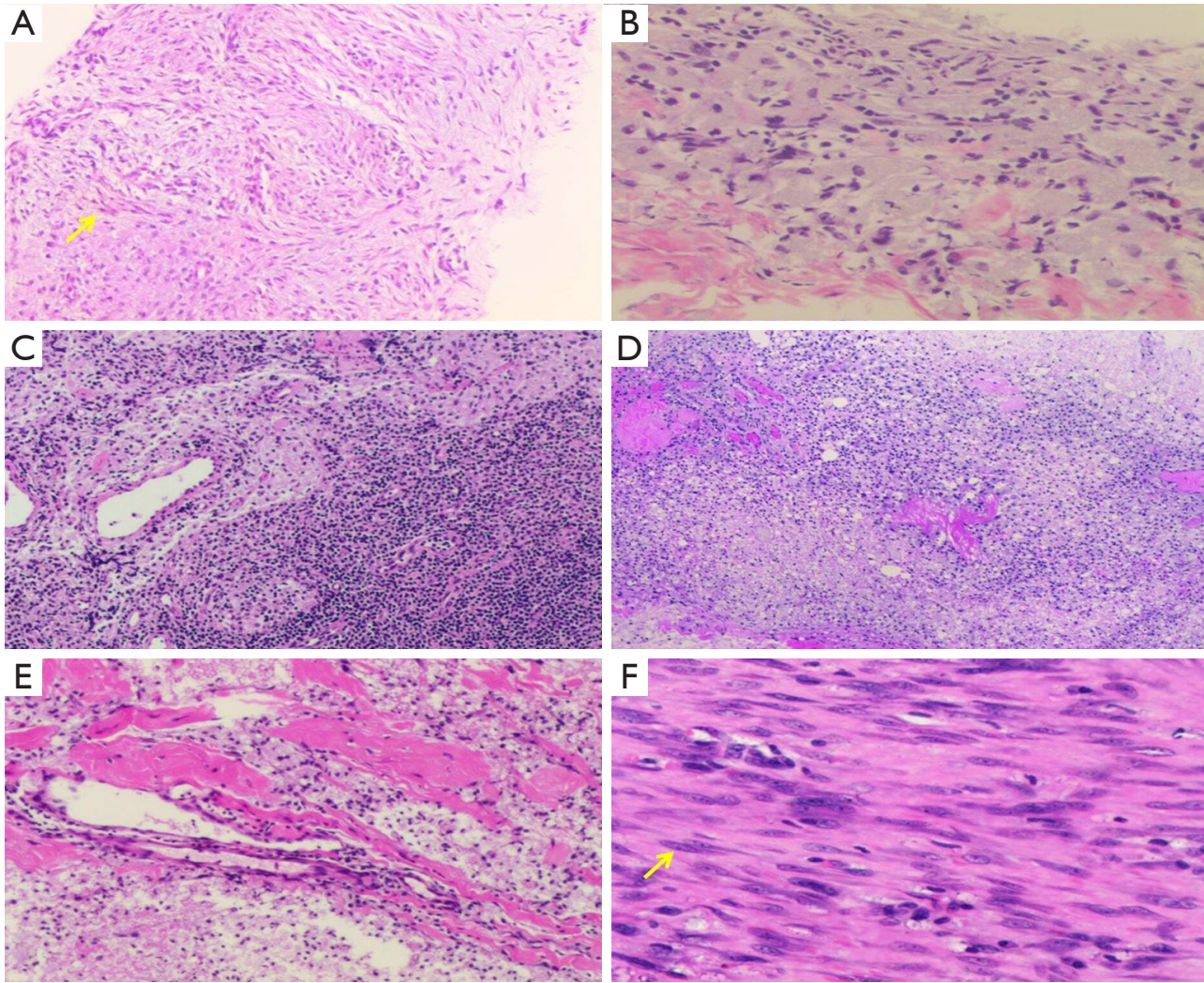

Figure $6 \mathrm{H} \& \mathrm{E}$ staining of specimens in the case. (A) $\times 40$, Bundles of spindle cells (arrow) were observed in mammary biopsy images; (B) $\times 100$, no tumor cell but some foam cells were observed in axillary biopsy image; (C) $\times 40$, sentinel lymph node and (D) $\times 40$, axillary lymph nodes were free from tumor invasion; $(\mathrm{E}) \times 100$, postoperative axillary pathology showed no malignancy but a large number of foam cells and chronic inflammatory cells; (F) $\times 400$, post-operative mammary pathology showed mildly atypical spindle cells (arrow) with mitotic figures counted 6/10 HPF.

new cases per 1 million individuals per year, and it rarely occurs in the breast, accounting for fewer than $10 \%$ of all aggressive fibromatosis and less than $0.2 \%$ of all primary breast cancer $(6,10)$. According to the 5 th edition of the WHO Classification of Tumours of the Breast, aggressive breast fibromatosis includes tumor from the breast parenchyma and tumor from the pectoral muscle. The Wnt/ $\beta$-catenin pathway appears to play a key role in aggressive fibromatosis pathogenesis, and recent studies suggest a possible model of its pathogenesis. Another study has reported that mutations in APC gene have a high incidence of aggressive fibromatosis. In aggressive fibromatosis tumor, the main role of $\beta$-catenin is being part of the transcription apparatus in the nucleus. Intracellular levels of $\beta$-catenin are controlled by adenomatous polyposis coli (APC) complex that phosphorylates $\beta$-catenin, inducing its destruction by the proteasome. In normal physiology, this process is inhibited by activation of the Wnt pathway, which leads to inhibition of the kinase activity of the APC complex and causes non-phosphorylated $\beta$-catenin accumulates in the cytoplasm. This process facilitates cellular proliferation, survival, and differentiation (1113). This disease occurs in women aged between 13 and 80 years. Its etiology remains uncertain, but several cases reported aggressive fibromatosis was associating with previous mammary trauma, surgery, and hereditary diseases such as FAP (familial adenomatous polyposis). Though there is an underlying genetic-based association between the familial cases and FAP, the pathogenesis of FAP-associated cases is multifactorial, and another associated factor is trauma (usually surgery). Mutations of the APC/ $\beta$-catenin pathway might be responsible for the development of FAPassociated aggressive fibromatosis, and the location of the APC gene mutation is thought to influence morphologic of 

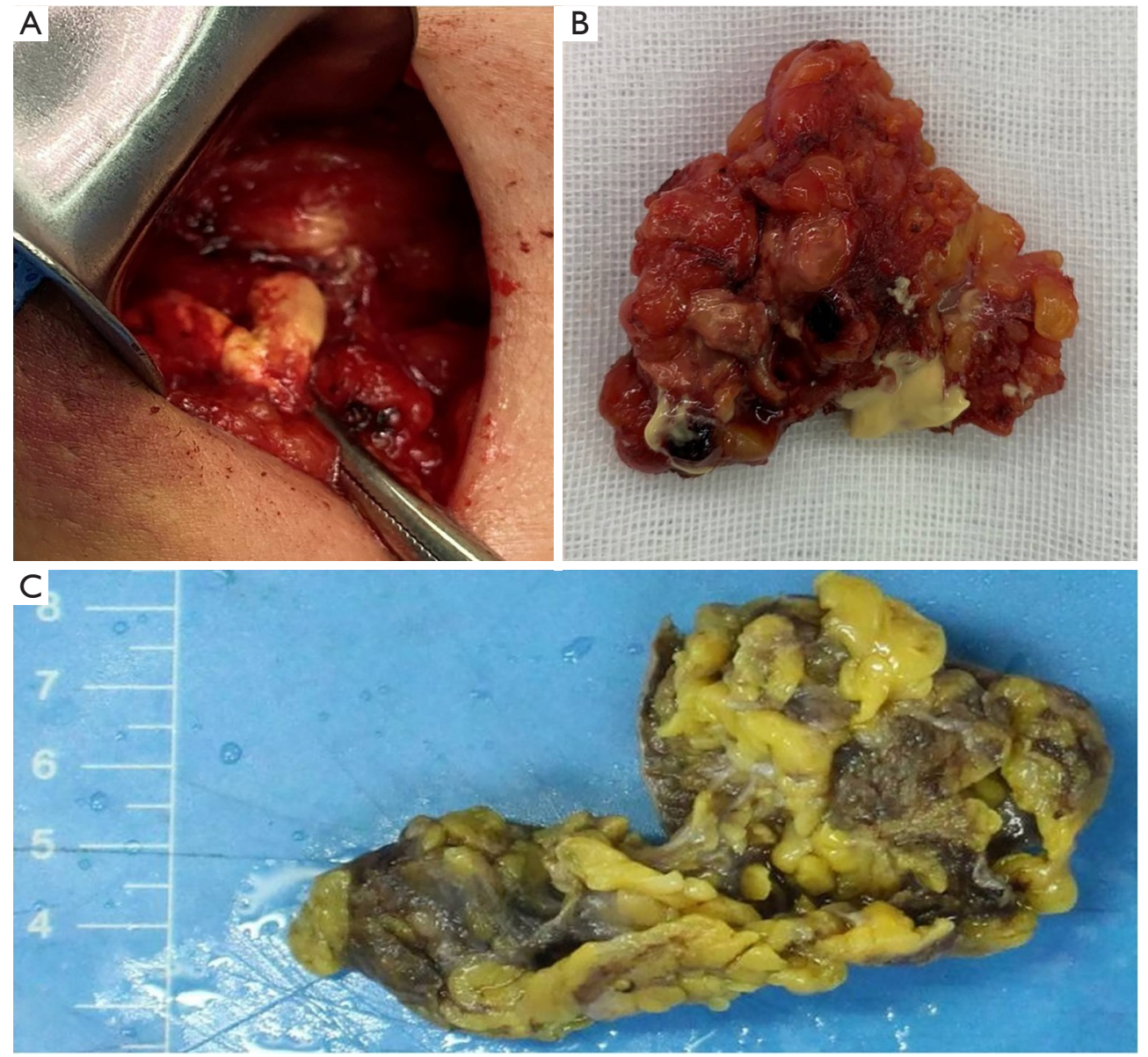

Figure 7 Pictures of the masses. (A) In the axillary area, caseous necrosis tissue were observed during surgery; (B) axillary gross specimen and (C) mammary surgical specimen.

the tumor $(1,11,13,14)$.

According to reported cases, the recurrence of aggressive breast fibromatosis mostly occurred within three years after surgical resection, and the local recurrence rate is $29 \%$ $(5,6)$. Prompt diagnosis of aggressive breast fibromatosis can be challenging especially when accompanying with axillary lesions. Clinically, aggressive breast fibromatosis usually presents as a palpable painless firm mass, commonly with skin retraction. However, nipple discharge or axillary lymphadenopathy is uncommon $(5,15)$. Our cases showed a unilateral mass with typical skin dimpling in physical examination, which mimics breast carcinoma. Radiologically, aggressive breast fibromatosis typically presents as an irregular spiculated mass on both mammograms and ultrasound, and the mass could have atypical sonographic and radiological features that mimic carcinoma (16). Breast MRI is a more sensitive method to assess aggressive fibromatosis, and define tumoral morphology and extent than mammograms and ultrasound. It is helpful for surgeons to determine the surgical region preoperatively to make sure the margins negative (17). Thus breast MRI is almost essential for assessing soft-tissue mass and guiding clinical treatment (18). $\mathrm{PET/CT}$ is not the necessary examination for aggressive fibromatosis, but it could provide more valuable information about the pathological metabolic activity and distant metastasis of tumor.

Mammography of our case presents no mass and no calcifications. However, the contrast-enhanced ultrasound revealed an irregular mass with unclear boundaries and abundant blood supply. Moreover, enlarged masses in the ipsilateral axilla were considered as axillary lymph nodes metastasis. DCE-MRI of our case revealed a $1.6 \mathrm{~cm} \times$ $1.7 \mathrm{~cm}$ irregular mass with an unclear margin to the adjacent 
chest wall, which was classified into BI-RADS 4c. However, the images demonstrated no enlarged mass in the axilla. The 18F-FDG PET/CT of our case revealed an axillary locally hypermetabolic lesion, and that was considered as lymph node metastasis. We listed some representative reported cases that are same or similar to our case in Table 1 (4-6,1531 ), and the sonographic and radiological manifestations of these cases could be compared intuitively to give helpful information for clinicians. Of the 24 cases listed, the clinical and imaging manifestations are various and the masses were usually misdiagnosed as carcinoma, Prior studies have reported that mammography, ultrasound and MRI were used extensively. The masses are most often spiculated in mammography and irregular and hypoechoic in ultrasound.

For aggressive breast fibromatosis, histopathological examination is the gold standard for final diagnosis. Coreneedle biopsy examination can provide a preoperative diagnosis and helps to make treatment options $(7,18)$. Microscopically, the vital feature of aggressive breast fibromatosis is the fascicular-arranged spindle cells without pathological mitosis. Minimal nuclear atypia of spindle cells may be seen in some cases. The tumor may infiltrate adjacent tissue in a finger-shaped form. The characteristic immunohistochemical biomarker of aggressive fibromatosis is $\beta$-catenin. However, nuclear immunohistochemical staining for $\beta$-catenin is not always positive in all cases, so the negative staining result cannot exclude aggressive fibromatosis (7). Other biomarkers, such as vimentin, desmin, and smooth muscle actin, are erratic to the definitive diagnosis of the disease (22). The negative expression of S-100 and CD34 is also essential to differentiate aggressive fibromatosis from another disease (7). The differential diagnosis of aggressive breast fibromatosis is challenging, includes neoplastic spindle cell proliferations, such as fibromatosislike spindle cell metaplastic carcinoma, cystosarcoma phylloides, fibrosarcoma and myofibroblastic tumor and benign lesions such as radial scars and nodular fasciitis. Immunohistochemistry staining including SMA, $\beta$-catenin (nuclear), CD34, S-100 protein and cytokeratins can help to make the differential diagnosis. The most important differential is with fibromatosis-like spindle cell metaplastic carcinoma, and nuclear atypia and an elevated mitotic rate could help to differentiate from aggressive fibromatosis. To distinguish aggressive fibromatosis from fibrosarcoma, the absence of nuclear pleomorphism and high mitotic index in microscopy are helpful. The opinion of a specialist soft tissue pathologist is useful in diagnosing such disease
$(1,8,9)$. In our case, the preoperative pathology supported aggressive breast fibromatosis. The postoperative gross pathology revealed the positive expression of $\beta$-catenin, the focal lower expression of CK, and the negative expression of p63, CD34, CK (HMW), and CK5/6. The definitive diagnosis was made that the mammary mass was aggressive breast fibromatosis, and the axillary nodules were accessory breast mastitis.

Standard treatment for aggressive breast fibromatosis has not been established until now. However, wide surgical resection seems to be the preferred approach for patients with such a disease $(4,32)$. It is crucial to ensure the negative margins during surgery, which is beneficial to avoid local recurrence. However, recurrence still occurs in $27 \%$ of cases who had negative margins after surgical excision (33). As the repeated surgical excision would destroy the appearance of the chest wall and increases patients' burdens, active observation has been applied as another option rather than surgery $(21,22)$. Nevertheless, there is no consensus in the identification of those patients who can be avoided from surgery (34). It is not negligible that more aggressive surgical resection would be performed for these patients if the disease has progressed during observation.

Clinically, postoperative radiotherapy has also been applied to reduce the risk of recurrence in patients with positive margins. Studies have reported that the rate of recurrence decreased from $59 \%$ to $25 \%$ in patients undergoing postoperative radiotherapy $(6,20)$. However, radiotherapy also causes some side effects such as local pain, appearance change, and skin toxicity. Studies have reported a significant effect of the combination of Tamoxifen and Celecoxib for a recurrent patient. Tamoxifen is helpful for stabilization of the residual tumor, sometimes even causes tumoral regression (6). But the optimal regime, dose, and duration of such a drug remain unclear because of limited clinical application to aggressive breast fibromatosis $(4,21,35)$. Literature has reported that the application of methotrexate and vincristine combination is helpful for patients who are inoperable $(18,36)$. Non-steroidal antiinflammatory drugs, tyrosine kinase inhibitors, hormones drugs, interferons, and cytotoxic drugs were all effective clinical protocols previously reported to help control recurrent aggressive breast fibromatosis $(4,32)$.

\section{Conclusions}

Aggressive breast fibromatosis is a rare tumor that is often locally aggressive and easy to recur postoperatively without 


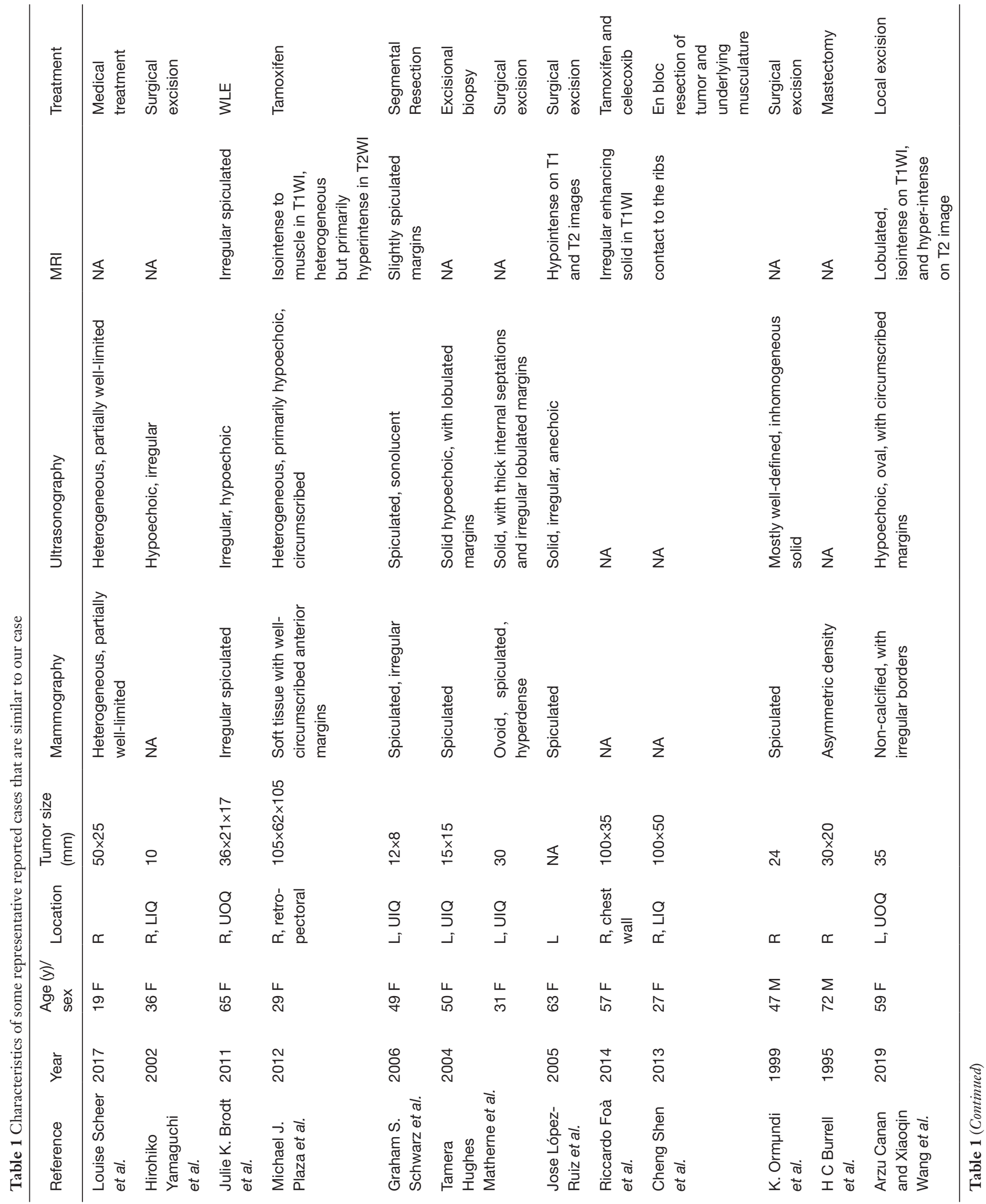




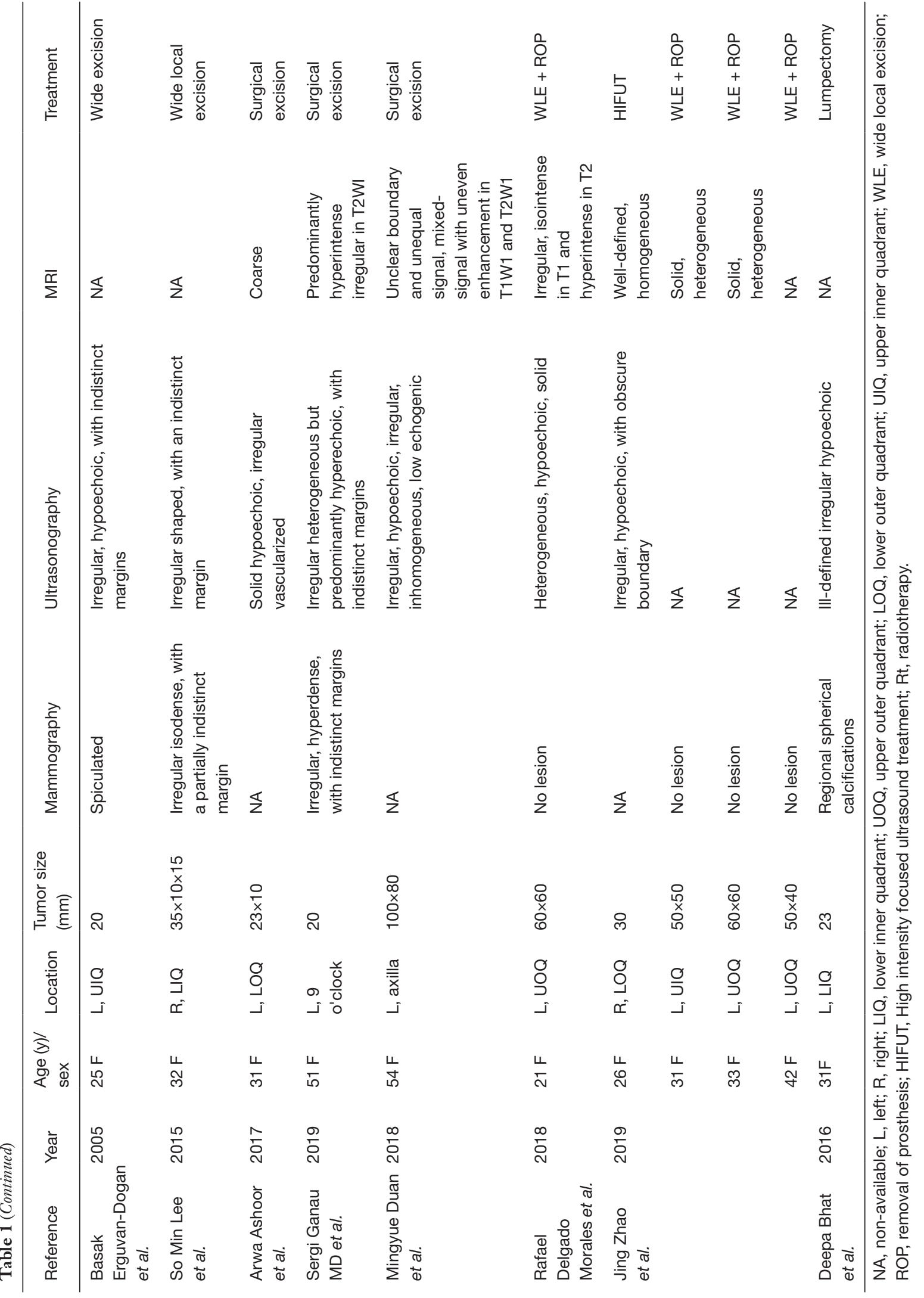


the potential of distant metastasis. It can be misdiagnosed as breast carcinoma due to similar clinical and imaging manifestation. MRI and preoperative biopsy are of vital importance to the accurate diagnosis. Optimal management of aggressive breast fibromatosis remains controversial, and multiple treatment options have been reported. Wide surgical excision with negative margins is the preferred approach to patients with aggressive breast fibromatosis as it can ensure long-term remission. Radiotherapy may be beneficial to postoperative patients by reducing the local recurrence rate. Up till now, there has not been a standardized clinical strategy to treat aggressive breast fibromatosis due to its low incidence and limited data. Further studies to such a disease are expected, and our case will contribute to improving our knowledge of this disease.

\section{Acknowledgments}

Funding: This study was supported by the Sun Yat-sen University Clinical Research 5010 Program (2016007).

\section{Footnote}

Reporting Checklist: The authors have completed the CARE checklist. Available at http://dx.doi.org/10.21037/tbcr-20-41

Conflicts of Interest: All authors have completed the ICMJE uniform disclosure form (available at http://dx.doi. org/10.21037/ tbcr-20-41). The authors have no conflicts of interest to declare.

Ethical Statement: The authors are accountable for all aspects of the work in ensuring that questions related to the accuracy or integrity of any part of the work are appropriately investigated and resolved. All procedures performed in studies involving human participants were in accordance with the ethical standards of the institutional and/or national research committee(s) and with the Helsinki Declaration (as revised in 2013). The patient has given the consent for the case report to be published.

Open Access Statement: This is an Open Access article distributed in accordance with the Creative Commons Attribution-NonCommercial-NoDerivs 4.0 International License (CC BY-NC-ND 4.0), which permits the noncommercial replication and distribution of the article with the strict proviso that no changes or edits are made and the original work is properly cited (including links to both the formal publication through the relevant DOI and the license). See: https://creativecommons.org/licenses/by-nc-nd/4.0/.

\section{References}

1. Glazebrook KN, Reynolds CA. Mammary fibromatosis. AJR Am J Roentgenol 2009;193:856-60.

2. Rosen Y, Papasozomenos SC, Gardner B. Fibromatosis of the breast. Cancer 1978;41:1409-13.

3. Tzur R, Silberstein E, Krieger Y, et al. Desmoid Tumor and Silicone Breast Implant Surgery: Is There Really a Connection? A Literature Review. Aesthetic Plast Surg 2018;42:59-63.

4. Scheer L, Lodi M, Molière S, et al. Medical treatment of mammary desmoid-type fibromatosis: which benefit? World J Surg Oncol 2017;15:86.

5. Brodt JK, Rhodes DJ, Glazebrook KN, et al. Radiologic and pathologic images of mammary fibromatosis. The breast journal 2011;17:207-9.

6. Plaza MJ, Yepes M. Breast fibromatosis response to tamoxifen: dynamic MRI findings and review of the current treatment options. J Radiol Case Rep 2012;6:16-23.

7. Ebrahim L, Parry J, Taylor DB. Fibromatosis of the breast: a pictorial review of the imaging and histopathology findings. Clin Radiol 2014;69:1077-83.

8. Dwyer JB, Clark BZ. Low-grade fibromatosis-like spindle cell carcinoma of the breast. Arch Pathol Lab Med 2015;139:552-7.

9. Ünal B, Erdoğan G, Karaveli F. Step by step approach to rare breast lesions containing spindle cells. Springerplus 2015;4:678.

10. Roussin S, Mazouni C, Rimareix F, et al. Toward a new strategy in desmoid of the breast? Eur J Surg Oncol 2015;41:571-6.

11. Escobar C, Munker R, Thomas JO, et al. Update on desmoid tumors. Ann Oncol 2012;23:562-9.

12. Nieuwenhuis MH, Lefevre JH, Bülow S, et al. Family history, surgery, and APC mutation are risk factors for desmoid tumors in familial adenomatous polyposis: an international cohort study. Dis Colon Rectum 2011;54:1229-34.

13. Abraham SC, Reynolds C, Lee JH, et al. Fibromatosis of the breast and mutations involving the APC/beta-catenin pathway. Hum Pathol 2002;33:39-46.

14. Nandakumar G, Morgan J, Silverberg D, et al. Familial polyposis coli: clinical manifestations, evaluation, management and treatment. Mt Sinai J Med 
2004;71:384-91.

15. Canan A, Wang X. Recurrent desmoid tumor arising from latissimus dorsi flap: A case report. Clin Imaging 2019;53:191-4.

16. Ashoor A, Monti S, Pezzella M. Fibromatosis, a benign breast disease mimicking carcinoma. A case report. Int J Surg Case Rep 2017;41:392-7.

17. Ganau S, Frigola G, Bargalló X, et al. Breast fibromatosis: Variability among imaging methods. Breast J 2019;25:750-2.

18. Duan M, Xing H, Wang K, et al. A large and aggressive fibromatosis in the axilla: a rare case report and review of the literature. Onco Targets Ther 2018;11:3179-84.

19. Schwarz GS, Drotman M, Rosenblatt R, et al. Fibromatosis of the breast: case report and current concepts in the management of an uncommon lesion. Breast J 2006;12:66-71.

20. Bhat D, Wear V, Weisenberg E, et al. Desmoid-type fibromatosis of the breast: A case report. Breast Dis 2016;36:149-52.

21. Zhao J, Wang Q, Li X, et al. Effective high intensity focused ultrasound treatment in recurrent aggressive breast fibromatosis: a case report. Onco Targets Ther 2019;12:5251-6.

22. Morales RD, Mendoza AG, Luces C, et al. Aggressive breast fibromatosis following augmentation mastoplasty: a series of case reports. Ecancermedicalscience 2018;12:833.

23. Yamaguchi H, Sakakibara T, Hino M, et al. A case of fibromatosis of the breast. Breast Cancer 2002;9:175-8.

24. Matherne TH, Green A Jr, Tucker JA, et al. Fibromatosis: the breast cancer imitator. South Med J 2004;97:1100-3.

25. López-Ruiz J, Ruiz M, Echevarria JJ, et al. Mammary fibromatosis mimicking recurrent breast cancer: radiological findings. Eur Radiol 2005;15:2034-6.

26. Foà R, Rizzo S, Petrella F, et al. Recurrent aggressive fibromatosis of the chest wall. Ecancermedicalscience 2014;8:464.

doi: $10.21037 /$ tbcr-20-41

Cite this article as: Huang $\mathrm{Y}$, Shao N, Shi H, Zhang $\mathrm{X}$, Zheng Y, Lin Y. Aggressive breast fibromatosis with axillary accessory breast mastitis mimicking carcinoma: a case report. Transl Breast Cancer Res 2021;2:8.
27. Shen C, Zhou Y, Che G. Management of a female with recurrence of fibromatosis of the chest wall adjacent to the breast: a case report. J Cardiothorac Surg 2013;8:41.

28. Ormándi K, Lázár G, Tószegi A, et al. Extra-abdominal desmoid mimicking malignant male breast tumor. Eur Radiol 1999;9:1120-2.

29. Burrell HC, Sibbering DM, Wilson AR. Case report: fibromatosis of the breast in a male patient. Br J Radiol 1995;68:1128-9.

30. Erguvan-Dogan B, Dempsey PJ, Ayyar G, et al. Primary desmoid tumor (extraabdominal fibromatosis) of the breast. AJR Am J Roentgenol 2005;185:488-9.

31. Lee SM, Lee JY, Lee BH, et al. Fibromatosis of the breast mimicking an abscess: case report of unusual sonographic features. Clin Imaging 2015;39:685-8.

32. Skubitz KM. Biology and Treatment of Aggressive Fibromatosis or Desmoid Tumor. Mayo Clin Proc 2017;92:947-64.

33. Ballo MT, Zagars GK, Pollack A, et al. Desmoid tumor: prognostic factors and outcome after surgery, radiation therapy, or combined surgery and radiation therapy. J Clin Oncol 1999;17:158-67.

34. Kasper B, Baumgarten C, Garcia J, et al. An update on the management of sporadic desmoid-type fibromatosis: a European Consensus Initiative between Sarcoma PAtients EuroNet (SPAEN) and European Organization for Research and Treatment of Cancer (EORTC)/Soft Tissue and Bone Sarcoma Group (STBSG). Ann Oncol 2017;28:2399-408.

35. Bates JE, Morris CG, Iovino NM, et al. Radiation Therapy for Aggressive Fibromatosis: The Association Between Local Control and Age. Int J Radiat Oncol Biol Phys 2018;100:997-1003.

36. Park KH, Choi YJ, Kim KW, et al. Combination chemotherapy with methotrexate and vinblastine for surgically unresectable, aggressive fibromatosis. Jpn J Clin Oncol 2016;46:845-9. 